DOI (Digital Object Identifier) 10.1007/s102310100027

Barbara Bianconi · Matteo Focardi · Elvira Mascolo

\title{
Existence of minimizers for a class of quasi-convex functionals with non-standard growth
}

Received: June 14, 2000; in final form: November 25, 2000

Published online: December 19, 2001 - (C) Springer-Verlag 2001

\begin{abstract}
We study the lower semicontinuity properties of non-autonomous variational integrals whose energy densities satisfy general growth conditions. We apply these results to solve Dirichlet's boundary value problems for such functionals.
\end{abstract}

Mathematics Subject Classification (2000). 49J45

\section{Introduction}

In this paper we consider the variational approach to prove the existence of an equilibrium solution in non-linear elasticity. We take into account only elastic materials possessing stored energy functions, for such materials the problem consists in finding a vector field $u: \Omega \rightarrow \mathbb{R}^{N}$, where $\Omega$ is a bounded open subset of $\mathbb{R}^{n}$, which is a solution of

$$
m=\inf \left\{F(u, \Omega): u=u_{0} \text { on } \partial \Omega\right\},
$$

where

$$
F(u, \Omega)=\int_{\Omega} f(x, u(x), D u(x)) d x,
$$

with $f: \Omega \times \mathbb{R}^{N} \times \mathbb{R}^{N n} \rightarrow \mathbb{R}$ a Carathéodory's integrand. As usual, we study this problem by using the Direct Methods of the Calculus of Variations, therefore the main question is to determine the conditions on $f$ ensuring coercivity and sequential lower semicontinuity for $F(\cdot, \Omega)$ with respect to the same topology.

A suitable condition on stored energy function $f$, termed quasi-convexity, was introduced by Morrey in a fundamental paper in 1952: $f$ is quasi-convex in $z$ in the Morrey sense if, for every $\left(x_{0}, s_{0}, z_{0}\right) \in \Omega \times \mathbb{R}^{N} \times \mathbb{R}^{N n}$ and $\varphi \in C_{0}^{\infty}\left(\Omega, \mathbb{R}^{N}\right)$, there holds

$$
f\left(x_{0}, s_{0}, z_{0}\right) \mathcal{L}^{n}(\Omega) \leq \int_{\Omega} f\left(x_{0}, s_{0}, z_{0}+D \varphi(y)\right) d y
$$

B. Bianconi, M. Focardi, E. Mascolo: Dipartimento di Matematica "U. Dini" - Universitá di Firenze, Viale Morgagni 67/A - 50134 Firenze, Italy, e-mail: bianconi@math.unifi.it, mascolo@math.unifi.it, focardi@math.unifi.it 
denoting by $\mathcal{L}^{n}(\Omega)$ the $n$ dimensional Lebesgue's measure of $\Omega$. Morrey showed under strong regularity assumptions on $f$, that $F(\cdot, \Omega)$ is sequentially lower semicontinuous in the weak* topology of $W^{1, \infty}\left(\Omega, \mathbb{R}^{N}\right)$ if and only if $f$ is quasi-convex.

In recent years, great interest has been raised around quasi-convex integrals of type (1.2), satisfying the so-called $(p, q)$ growth conditions, i.e.,

$$
c_{0}\left(|z|^{p}-1\right) \leq f(x, s, z) \leq c_{1}\left(1+|z|^{q}\right),
$$

with $1 \leq p \leq q$. Indeed, in non-linear elasticity, conditions $N=n=q$ and $q>p$ play a fundamental role in the study of cavitation since they allow discontinuous deformations of the elastic body.

When $p=q$, the case of natural growth, Acerbi-Fusco [2] and Marcellini [32] proved the sequential weak lower semicontinuity of $F(\cdot, \Omega)$ in the weak topology of $W^{1, p}\left(\Omega, \mathbb{R}^{N}\right)$.

Many authors have studied the lower semicontinuity and relaxation properties for functionals satisfying (1.3) in the Sobolev space setting, obtaining sharp conditions on the mutual dependence of $p$ and $q$. When $f=f(x, z) \geq 0$, and imposing further structure conditions on $f$, the lower semicontinuity inequality

$$
\liminf _{r} F\left(u_{r}, \Omega\right) \geq F(u, \Omega)
$$

has been established by Marcellini [33] along sequences $\left(u_{r}\right)$ in $W^{1, q}\left(\Omega, \mathbb{R}^{N}\right)$ converging in the weak topology of $W^{1, p}\left(\Omega, \mathbb{R}^{N}\right)$ for $p>\frac{n}{n+1} q$. In the autonomous case $f=f(z)$ the lower semicontinuity inequality (1.4) was proven to hold true for $p>q-1$ by Fonseca-Marcellini [18], and for $p>\frac{n-1}{n} q$ by Fonseca-Malý [17]. See also Malý [30] for related counterexamples and others for refinements (see [1], [5], [11], [22], [23], [27], [29], [31]).

However, this approach cannot be directly applied to establish existence results of Dirichlet's boundary value problems since the different topologies with respect to whom the functionals are coercive and lower semicontinuous.

Our aim is to study a particular class of integrands with $(p, q)$ growth, those for which the stored energy function is controlled in terms of suitable convex functions. More precisely, we assume that $f$ is a quasi-convex function satisfying the non-standard growth conditions

$$
\left.-c_{1} \Phi_{1}(|z|)-c_{2} \Phi_{2}(|s|)-c_{3}(x) \leq f(x, s, z) \leq g(x, s)\{1+\Phi(\mid z))\right\},
$$

where $c_{1}, c_{2}$ are positive constants; $c_{3} \in L^{1}(\Omega) ; \Phi, \Phi_{1}$ and $\Phi_{2}$ are $N$-functions suitably related; and $g: \Omega \times \mathbb{R}^{N} \rightarrow \mathbb{R}$ is a positive Carathéodory function.

With these general growth conditions, Orlicz-Sobolev spaces provide the natural setting to study the lower semicontinuity properties of functionals in (1.2). Indeed we prove that $F(\cdot, \Omega)$ is sequentially lower semicontinuous in the weak* topology of the Orlicz-Sobolev space $W^{1} L^{\Phi}\left(\Omega, \mathbb{R}^{N}\right)$, assuming that in (1.5) $\Phi$ satisfying a sub-homogeneity property at infinity called $\Delta_{2}$ property, and $\Phi_{1}, \Phi_{2}$ satisfying some asymptotic conditions with respect to $\Phi$.

Moreover, we estabilish an existence result for such a class of integrands. Thus, we are able to study energy densities, depending on the full set of the variables 
with $(p, q)$ growth and oscillating behaviour. Indeed, the coercivity and lower semicontinuity now holds in the Orlicz-Sobolev spaces setting.

Ball [4] was the first to set some variational problems in the framework of Orlicz-Sobolev spaces considering the poly-convex case. Recently, Focardi in [15] has proved the lower semicontinuity properties for functionals in (1.2) in OrliczSobolev spaces for the integrands $f=f(z)$ satisfying the non-standard growth conditions (1.5) with $\Phi \in \Delta_{2}$. This result will be an ingredient in proving the semicontinuity Theorem 3.1 below.

The case of $N$-functions, $\Phi$, not sharing the $\Delta_{2}$ property, corresponding roughly to exponential growth, has been considered by Focardi-Mascolo in [16] where a suitable semicontinuity property has been proved.

The plan of the paper is the following:

Section 2 is devoted to all the preliminary results about Orlicz-Sobolev spaces and the quasi-convex envelope of a function; moreover, we recall the statement of a recent result of higher integrability for local minimizers of integral functionals with general growth conditions proved by Cianchi-Fusco [7]. In Sect. 3 we prove a semicontinuity result, obtained by suitable modifications of the arguments used in the natural growth case by Marcellini [32]. The proof is based on an approximation procedure of the stored energy function by a non-decreasing sequence of quasiconvex functions. Eventually, Sect. 4 is devoted to the proof of an existence theorem and to some application to non-trivial examples.

\section{Notations and preliminaries}

We denote by $\langle\cdot, \cdot\rangle$ the Euclidean scalar product in $\mathbb{R}^{n}$ and with $|\cdot|$ the usual Euclidean norm. Throughout all the paper $\Omega$ denotes an open and bounded subset of $\mathbb{R}^{n}$ with Lipschitz boundary. We denote by $\mathcal{L}^{n}$ the Lebesgue measure on $\mathbb{R}^{n}$ and the notation a.e. stands for almost everywhere with respect to Lebesgue measure. We use standard notations for spaces of classically differentiable functions, Lebesgue and Sobolev spaces. Given any function $u \in L^{1}(\Omega)$ the symbol $f_{\Omega} u d x$ stands for the average of $u$ over $\Omega$, i.e., $\frac{1}{\alpha^{n}(\Omega)} \int_{\Omega} u d x$.

\subsection{N-functions and Orlicz spaces}

For ease of reference we recall some definitions and known properties of $\mathrm{N}$ functions and Orlicz spaces (see [26], [35]).

A convex function $\Phi:[0,+\infty[\rightarrow[0,+\infty[$ is called an $N$-function if it satisfies the following conditions: $\Phi(0)=0, \Phi(t)>0$ for $t>0$, and

$$
\lim _{t \rightarrow 0} \frac{\Phi(t)}{t}=0, \quad \lim _{t \rightarrow+\infty} \frac{\Phi(t)}{t}=+\infty .
$$

Such a function $\Phi$ has an integral representation of the form

$$
\Phi(t)=\int_{0}^{t} p(s) d s
$$


for every $t \geq 0$, where $p:[0,+\infty[\rightarrow[0,+\infty[$ is non-decreasing, right continuous and it satisfies the conditions: $p(0)=0, p(s)>0$ for $s>0$, and

$$
\lim _{s \rightarrow+\infty} p(s)=+\infty \text {. }
$$

The function $p$ is called the right derivative of $\Phi$.

The notion of an $\mathrm{N}$-function can be relaxed, in the sense that only the behaviour at infinity is important. Indeed, given any convex function $Q:[0,+\infty[\rightarrow$ $[0,+\infty[$, satisfying

$$
\lim _{t \rightarrow+\infty} \frac{Q(t)}{t}=+\infty
$$

there exists an $\mathrm{N}$-function $\Phi$ and $t_{0} \geq 0$ such that $Q(t)=\Phi(t)$ for every $t \geq t_{0}$. Such a function $Q$ is called the principal part of the $\mathrm{N}$-function $\Phi$, because of this we will not distinguish between the two concepts any longer.

The set of N-functions can be endowed with a partial ordering, we say that $\Phi_{1}$ dominates $\Phi_{2}$, and we write $\Phi_{2} \prec \Phi_{1}$, if there exist two constants $k, t_{0}>0$ such that, for every $t \geq t_{0}$, it holds

$$
\Phi_{2}(t) \leq \Phi_{1}(k t) .
$$

If, moreover, $\Phi_{2} \prec \Phi_{1}$ and $\Phi_{1} \prec \Phi_{2}$ we say that $\Phi_{1}$ and $\Phi_{2}$ are equivalent, while if $\Phi_{1}$ dominates $\Phi_{2}$ but $\Phi_{1}, \Phi_{2}$ are not equivalent we say that $\Phi_{1}$ dominates strictly $\Phi_{2}$, and we write $\Phi_{2} \prec \prec \Phi_{1}$. We remark that if $\Phi_{2} \prec \prec \Phi_{1}$ there exists an $\mathrm{N}$-function $\Gamma$ such that $\Gamma \circ \Phi_{2} \prec \Phi_{1}$. For instance, $\Gamma$ can be defined as the primitive of

$$
q(s)= \begin{cases}\inf \left\{\frac{\Phi_{1}\left(\Phi_{2}^{-1}(t)\right)}{t}: t>s\right\} & s \geq 1 \\ q(1) s & 0 \leq s<1 .\end{cases}
$$

Let $\Phi$ be an $\mathrm{N}$-function, define the function

$$
\widetilde{\Phi}(t)=\max _{s>0}\{s t-\Phi(s)\},
$$

$\widetilde{\Phi}$ is an N-function called the complementary $N$-function of $\Phi$. By the very definition of $\widetilde{\Phi}$, the pair $\Phi, \widetilde{\Phi}$ satisfies Young's inequality, i.e.,

$$
s t \leq \Phi(s)+\widetilde{\Phi}(t)
$$

for every $s, t \geq 0$, with equality holding if $t=p(s)$ or $s=\tilde{p}(t)$, where $\tilde{p}$ is the right derivative of $\widetilde{\Phi}$.

In the following we will consider a special class of $\mathrm{N}$-functions.

Definition 2.1. We say that an $N$-function, $\Phi$, satisfies the $\Delta_{2}$ condition, and we write $\Phi \in \Delta_{2}$, if there exist two constants $k>1$ and $t_{0} \geq 0$ such that for every $t \geq t_{0}$, there holds

$$
\Phi(2 t) \leq k \Phi(t) .
$$


By taking into account Proposition 2.1 of [10] we infer the following result:

Proposition 2.2. Let $\Phi$ be an $N$-function, the following conditions are equivalent:

(i) $\Phi \in \Delta_{2}$;

(ii) there exists $r>1$ and $t_{0} \geq 0$ such that for every $t \geq t_{0}$ there holds

$$
t p(t) \leq r \Phi(t)
$$

(iii) there exists $r>1$ and $t_{0} \geq 0$ such that for every $t \geq t_{0}$ and $\lambda>1$ there holds

$$
\Phi(\lambda t) \leq \lambda^{r} \Phi(t)
$$

Conditions (ii) and (iii) above hold true with the same $r>1$, hence we write $\Phi \in \Delta_{2}^{r}$. It is easy to check that $\Phi(t)=t^{r}$ belongs to $\Delta_{2}^{r}, r>1$, and that $\Phi(t)=t^{r} \log ^{\alpha}(1+t)$, for $r \geq 1$ and $\alpha>0$, is an N-function of class $\Delta_{2}^{r+\varepsilon}$ for every $\varepsilon>0$. Moreover, the functions $\Phi(t)=\frac{t^{r}}{\log (1+t)}$, with $r \geq \frac{3}{2}$, and $\Phi(t)=t^{a+b \sin (\sin (\log (t)))}$, with $a>1+b \sqrt{2}$, are N-functions of class $\Delta_{2}$. The function $\Phi(t)=e^{t}-t-1$ is an $\mathrm{N}$-function which is not in class $\Delta_{2}$ (for further properties of $\mathrm{N}$-functions of class $\Delta_{2}$ see [3], [26], [28], [35]).

Let $\Omega$ be an open bounded set of $\mathbb{R}^{n}$, the Orlicz class $K^{\Phi}\left(\Omega, \mathbb{R}^{N}\right)$ is the set of all (equivalence classes modulo equality a.e. in $\Omega$ of) measurable functions $u: \Omega \rightarrow \mathbb{R}^{N}$ satisfying

$$
\int_{\Omega} \Phi(|u|) d x<+\infty
$$

The Orlicz space $L^{\Phi}\left(\Omega, \mathbb{R}^{N}\right)$ is defined to be the linear hull of $K^{\Phi}\left(\Omega, \mathbb{R}^{N}\right)$. The functional $\|\cdot\|_{\Phi, \Omega}: L^{\Phi}\left(\Omega, \mathbb{R}^{N}\right) \rightarrow \mathbb{R}$, defined by

$$
\|u\|_{\Phi, \Omega}=\inf \left\{\lambda>0: \int_{\Omega} \Phi\left(\frac{|u|}{\lambda}\right) d x \leq 1\right\},
$$

is a norm, called the Luxemburg norm, and $L^{\Phi}\left(\Omega, \mathbb{R}^{N}\right)$ is a Banach space if endowed with it. In the following we will denote by $s-L^{\Phi}\left(\Omega, \mathbb{R}^{N}\right)$ the norm convergence in $L^{\Phi}\left(\Omega, \mathbb{R}^{N}\right)$.

The closure of $C_{0}^{\infty}\left(\Omega, \mathbb{R}^{N}\right)$ in the norm topology of $L^{\Phi}\left(\Omega, \mathbb{R}^{N}\right)$ is denoted by $E^{\Phi}\left(\Omega, \mathbb{R}^{N}\right)$, the inclusions $E^{\Phi}\left(\Omega, \mathbb{R}^{N}\right) \subseteq K^{\Phi}\left(\Omega, \mathbb{R}^{N}\right) \subseteq L^{\Phi}\left(\Omega, \mathbb{R}^{N}\right)$ are trivial with equalities holding if and only if $\Phi \in \Delta_{2}$.

The following result on the integral convergence in Orlicz spaces has been proved in [16].

Proposition 2.3. Let $u \in E^{\Phi}\left(\Omega, \mathbb{R}^{N}\right)$, then for every $\left(u_{r}\right) \rightarrow$ u in $s-L^{\Phi}\left(\Omega, \mathbb{R}^{N}\right)$ and for every $\lambda>0$ there holds

$$
\lim _{r} \int_{\Omega} \Phi\left(\lambda\left|u_{r}\right|\right) d x=\int_{\Omega} \Phi(\lambda|u|) d x .
$$

The partial ordering introduced in the set of $\mathrm{N}$-functions induces topological embeddings among Orlicz spaces. 
Proposition 2.4. Let $\Phi_{1}, \Phi_{2}$ be two $N$-functions such that $\Phi_{2} \prec \Phi_{1}$, then the embedding

$$
L^{\Phi_{1}}\left(\Omega, \mathbb{R}^{N}\right) \hookrightarrow L^{\Phi_{2}}\left(\Omega, \mathbb{R}^{N}\right)
$$

is continuous. Moreover, if $\Phi_{2} \prec \prec \Phi_{1}$ then

$$
L^{\Phi_{1}}\left(\Omega, \mathbb{R}^{N}\right) \hookrightarrow E^{\Phi_{2}}\left(\Omega, \mathbb{R}^{N}\right) .
$$

The Orlicz-Sobolev space $W^{1} L^{\Phi}\left(\Omega, \mathbb{R}^{N}\right)$ is defined to be the set of all functions in $L^{\Phi}\left(\Omega, \mathbb{R}^{N}\right)$ whose first-order distributional derivatives are in $L^{\Phi}\left(\Omega, \mathbb{R}^{N}\right)$. It is a Banach space if endowed with the norm

$$
\|u\|_{1, \Phi, \Omega}=\|u\|_{\Phi, \Omega}+\|\nabla u\|_{\Phi, \Omega} .
$$

As in the case of ordinary Sobolev spaces, $W_{0}^{1} E^{\Phi}\left(\Omega, \mathbb{R}^{N}\right)$ is taken to be the closure of $C_{0}^{\infty}\left(\Omega, \mathbb{R}^{N}\right)$ in the norm of $W^{1} L^{\Phi}\left(\Omega, \mathbb{R}^{N}\right)$.

Let $\Phi$ be a given $\mathrm{N}$-function, we may suppose that

$$
\int_{0}^{1} \frac{\Phi^{-1}(s)}{s^{1+\frac{1}{n}}} d s<+\infty,
$$

replacing, if necessary, $\Phi$ by an equivalent $\mathrm{N}$-function. Assume, moreover, that

$$
\int_{1}^{+\infty} \frac{\Phi^{-1}(s)}{s^{1+\frac{1}{n}}} d s=+\infty,
$$

then we define the Sobolev's conjugate function $\Phi_{*}$ of $\Phi$ by

$$
\left(\Phi_{*}\right)^{-1}(t)=\int_{0}^{t} \frac{\Phi^{-1}(s)}{s^{1+\frac{1}{n}}} d s,
$$

for every $t \geq 0$. The following compact embedding theorem generalizes to OrliczSobolev spaces Rellich-Kondrakov's one (see Chap. VII of [3], [6] and Sect. 7.4 of [28]).

Theorem 2.5. Let $\Phi$ be an $N$-function.

(i) If (2.7) holds, the embedding

$$
W^{1} L^{\Phi}\left(\Omega, \mathbb{R}^{N}\right) \hookrightarrow L^{\Phi_{*}}\left(\Omega, \mathbb{R}^{N}\right)
$$

is continuous. Moreover, the embedding

$$
W^{1} L^{\Phi}\left(\Omega, \mathbb{R}^{N}\right) \hookrightarrow L^{\Phi_{1}}\left(\Omega, \mathbb{R}^{N}\right)
$$

is compact for every $N$-function $\Phi_{1} \prec \prec \Phi_{*}$.

(ii) If (2.7) does not hold, the embedding

$$
W^{1} L^{\Phi}\left(\Omega, \mathbb{R}^{N}\right) \hookrightarrow C^{0}\left(\bar{\Omega}, \mathbb{R}^{N}\right)
$$

is compact. 
We now introduce the weak $*$ convergence in $L^{\Phi}\left(\Omega, \mathbb{R}^{N}\right)$, denoted by $* w-$ $L^{\Phi}\left(\Omega, \mathbb{R}^{N}\right)$. Since the Orlicz space $L^{\Phi}\left(\Omega, \mathbb{R}^{N}\right)$ is isometrically isomorphic to the dual space of $E^{\widetilde{\Phi}}\left(\Omega, \mathbb{R}^{N}\right)$, a sequence $u_{r} \rightarrow u * w-L^{\Phi}\left(\Omega, \mathbb{R}^{N}\right)$, if and only if for every $v \in E^{\widetilde{\Phi}}\left(\Omega, \mathbb{R}^{N}\right)$ there holds

$$
\lim _{r} \int_{\Omega}\left\langle u_{r}, v\right\rangle d x=\int_{\Omega}\langle u, v\rangle d x .
$$

Thus, by means of the Hahn-Banach theorem, we are able to characterize the weak $*$ convergence in $W^{1} L^{\Phi}\left(\Omega, \mathbb{R}^{N}\right)$, denoted by $* w-W^{1} L^{\Phi}\left(\Omega, \mathbb{R}^{N}\right)$, that is: $u_{r} \rightarrow u * w-W^{1} L^{\Phi}\left(\Omega, \mathbb{R}^{N}\right)$, if and only if $\left(u_{r}\right)$ and $\left(D_{i} u_{r}\right), 1 \leq i \leq n$, converge to $u$ and $D_{i} u * w-L^{\Phi}\left(\Omega, \mathbb{R}^{N}\right)$, respectively.

Following the notations of [13], [25], [36], $W_{0}^{1} L^{\Phi}\left(\Omega, \mathbb{R}^{N}\right)$ denotes the weak* closure of $W_{0}^{1} E^{\Phi}\left(\Omega, \mathbb{R}^{N}\right)$ in $W^{1} L^{\Phi}\left(\Omega, \mathbb{R}^{N}\right)$, hence the inclusion $W_{0}^{1} E^{\Phi}\left(\Omega, \mathbb{R}^{N}\right)$ $\subseteq W_{0}^{1} L^{\Phi}\left(\Omega, \mathbb{R}^{N}\right)$ is trivial. By taking into account Corollary 1.10 of [25], the intersection of $W_{0}^{1} L^{\Phi}\left(\Omega, \mathbb{R}^{N}\right)$ with $\prod_{i=1}^{n+1} E^{\Phi}\left(\Omega, \mathbb{R}^{N}\right)$ is exactly $W_{0}^{1} E^{\Phi}\left(\Omega, \mathbb{R}^{N}\right)$, therefore we can infer, when $\Phi \in \Delta_{2}$, the equality $W_{0}^{1} E^{\Phi}\left(\Omega, \mathbb{R}^{N}\right)=W_{0}^{1} L^{\Phi}\left(\Omega, \mathbb{R}^{N}\right)$.

\subsection{Quasi-convex envelope}

In this section we state the main properties of the quasi-convex envelope of a given measurable function satisfying non-standard growth conditions. The case of natural growth has been established by Dacorogna [8] (see also [24]). The generalization to the non-standard case is not difficult, we report only the statements of the results without the arguments of their proofs.

Let $h: \mathbb{R}^{N n} \rightarrow \mathbb{R}^{+}$be a measurable function, define

$$
\gamma_{\Omega}(z)=\inf \left\{f_{\Omega} h(z+D \varphi(y)) d y: \varphi \in C_{0}^{\infty}\left(\Omega, \mathbb{R}^{N}\right)\right\},
$$

by taking into account Proposition 5.3 of [24] we have that if $\Omega_{1}$ and $\Omega_{2}$ are bounded open sets of $\mathbb{R}^{n}$ then $\gamma_{\Omega_{1}} \equiv \gamma_{\Omega_{2}} \equiv \gamma_{\Omega}$, so that we can drop the dependence on $\Omega$ in the definition of $\gamma_{\Omega}$ and denote it just by $\gamma$.

Moreover, assume that:

(i) there exists an $\mathrm{N}$-function $\Phi \in \Delta_{2}$ such that for every $z \in \mathbb{R}^{N n}$

$$
c_{0} \Phi(|z|) \leq h(z) \leq c_{1}(1+\Phi(|z|)) ;
$$

(ii) there exists $w \in C^{0}\left(\mathbb{R}^{+}, \mathbb{R}^{+}\right)$, with $w(0)=0$, such that

$$
|h(z)-h(w)| \leq c(1+\Phi(|z|+|w|+1)) w(|z-w|) .
$$

By taking into account the growth condition (2.8) and the continuity assumption (2.9) it is easy to check that

$$
\gamma(z)=\inf \left\{f_{\Omega} h(z+D \varphi(y)) d y: \varphi \in W_{0}^{1} E^{\Phi}\left(\Omega, \mathbb{R}^{N}\right)\right\} .
$$

Moreover, the same assumptions imply that $\gamma$ is a continuous function. 
Define $Q_{c} h$, the quasi-convex envelope of $h$, to be

$$
Q_{c} h=\sup \{\phi: \phi \leq h \text { quasi-convex }\},
$$

then, arguing as in the case of natural growth we can prove the following characterization of $Q_{c} h$ :

Theorem 2.6. Let $h: \mathbb{R}^{N n} \rightarrow \mathbb{R}^{+}$satisfy (2.8) and (2.9), then $\gamma \equiv Q_{c} h$.

\subsection{A regularity result}

In the proof of Theorem 3.1 below we will need a regularity result for local minimizers of functionals with non-standard growth. In particular, we will make use of higher integrability properties recently proved by Cianchi-Fusco [7].

Let $h: \mathbb{R}^{N n} \rightarrow \mathbb{R}$ be a continuous function such that there exists an $\mathrm{N}$-function $\Phi \in \Delta_{2}$ for which

$$
c_{0} \Phi(|z|) \leq h(z) \leq c_{1}(1+\Phi(|z|))
$$

for every $z \in \mathbb{R}^{N n}$.

We say that $u$ is a quasi-minimum, or equivalently $Q$-minimum, for the functional $H: W^{1} L^{\Phi}\left(\Omega, \mathbb{R}^{N}\right) \rightarrow \mathbb{R}$ defined by

$$
H(v, \Omega)=\int_{\Omega} h(D v(x)) d x,
$$

if there exists a constant $Q$ such that for every open set $\Omega_{0} \subset \subset \Omega$ there hold

$$
\int_{\Omega_{0}} \Phi(|D u|) d x<+\infty
$$

and

$$
H\left(u, \Omega_{0}\right) \leq Q H\left(u+\psi, \Omega_{0}\right),
$$

for every weakly differentiable function $\psi: \Omega_{0} \rightarrow \mathbb{R}^{N}$ with compact support and such that $\int_{\Omega_{0}} \Phi(|D \psi|) d x<+\infty$.

The following result holds true (see Theorem 1.1 of [7]):

Theorem 2.7. Let $u$ be a $Q$-minimum of $H$ with $h$ as above, then for every open subset $\Omega_{0} \subset \subset \Omega$ there exists $\delta>0$ depending on $n, \Phi, \Omega_{0}, \operatorname{dist}\left(\Omega_{0}, \partial \Omega\right)$ and $\int_{\Omega_{0}} \Phi(|D u|) d x$ such that

$$
\int_{\Omega_{0}} \Phi(|D u|)\left(\frac{\Phi(|D u|)}{|D u|}\right)^{\delta} d x<+\infty .
$$

We remark that by using the same arguments as in [7], it is possible to prove that, given a sequence $\left(u_{k}\right)$ of $Q$-minima for $H(\cdot, \Omega)$ such that 


$$
\sup _{k} \int_{\Omega_{0}} \Phi\left(\left|D u_{k}\right|\right) d x<+\infty,
$$

with fixed $\Omega_{0} \subset \subset \Omega$, there exists a positive constant $\delta$ independent of $k$ such that

$$
\sup _{k} \int_{\Omega_{0}} \Phi\left(\left|D u_{k}\right|\right)\left(\frac{\Phi\left(\left|D u_{k}\right|\right)}{\left|D u_{k}\right|}\right)^{\delta} d x<+\infty .
$$

\section{A semicontinuity result}

Let $f: \Omega \times \mathbb{R}^{N} \times \mathbb{R}^{N n} \rightarrow \mathbb{R}$ be a Carathéodory function, i.e., $f$ is measurable with respect to $x$ for every $(s, z) \in \mathbb{R}^{N} \times \mathbb{R}^{N n}$ and continuous with respect to $(s, z)$ a.e. in $\Omega$, satisfying the growth condition

$$
-c_{1} \Phi_{1}(|z|)-c_{2} \Phi_{2}(|s|)-c_{3}(x) \leq f(x, s, z) \leq g(x, s)(1+\Phi(|z|)),
$$

where $c_{1}, c_{2}$ are positive constants, $c_{3} \in L^{1}(\Omega), \Phi$ is an $\mathrm{N}$-function of class $\Delta_{2}^{r}, \Phi_{1}$ and $\Phi_{2}$ are $\mathrm{N}$-functions such that $\Phi_{1} \prec \prec \Phi$ and either $\Phi_{2} \prec \prec \Phi_{*}$ if (2.7) holds or $\Phi_{2}$ is arbitrarily chosen otherwise and $g: \Omega \times \mathbb{R}^{N} \rightarrow \mathbb{R}$ is a positive Carathéodory function.

Assume that $f$ is quasi-convex with respect to $z$, i.e., for every $\left(x_{0}, s_{0}, z_{0}\right) \in$ $\Omega \times \mathbb{R}^{N} \times \mathbb{R}^{N n}$ and $\varphi \in C_{0}^{\infty}\left(\Omega, \mathbb{R}^{N}\right)$ there holds

$$
f\left(x_{0}, s_{0}, z_{0}\right) \leq f_{\Omega} f\left(x_{0}, s_{0}, z_{0}+D \varphi(y)\right) d y .
$$

The following semicontinuity theorem holds true:

Theorem 3.1. Let $f: \Omega \times \mathbb{R}^{N} \times \mathbb{R}^{N n} \rightarrow \mathbb{R}$ be as above, then the functional $F: W^{1} L^{\Phi}\left(\Omega, \mathbb{R}^{N}\right) \rightarrow \mathbb{R}$ defined by

$$
F(u, \Omega)=\int_{\Omega} f(x, u(x), D u(x)) d x
$$

is sequentially lower semicontinuous with respect to $* w-W^{1} L^{\Phi}\left(\Omega, \mathbb{R}^{N}\right)$.

The proof of Theorem 3.1 is based on the following approximation result:

Theorem 3.2. Let $f: \Omega \times \mathbb{R}^{N} \times \mathbb{R}^{N n} \rightarrow \mathbb{R}$ be as above, assume that (3.11) is substituted by

$$
c_{0} \Phi(|z|) \leq f(x, s, z) \leq g(x, s)(1+\Phi(|z|)),
$$

where $c_{0}$ is a positive constant.

Then there exists a sequence ( $\left.f_{k}\right)$ of Carathéodory functions quasi-convex with respect to $z$ such that $f_{k}: \Omega \times \mathbb{R}^{N} \times \mathbb{R}^{N n} \rightarrow \mathbb{R}$ satisfies

$$
\begin{gathered}
c_{0} \Phi(|z|) \leq f_{k}(x, s, z) \leq k(1+\Phi(|z|)), \\
f_{k}(x, s, z)=c_{0} \Phi(|z|) \quad|s| \geq k, \quad|z| \geq k, \\
f_{k} \leq f_{k+1}, \quad \sup _{k} f_{k}=f .
\end{gathered}
$$


Proof. In the first part of the proof the arguments are similar to those of Theorem 1.2 of [32]. However, for the sake of completeness we outline the main ideas.

First we perform a truncation with respect to variables $(x, s)$. For $i \in \mathbb{N}$, let $\phi_{i}: \mathbb{R} \rightarrow \mathbb{R}$ be a continuous function such that

$$
\phi_{i}(t)=1 \quad 0 \leq t \leq i-1, \quad \phi_{i}(t)=0 \quad t \geq i,
$$

set

$$
\eta_{i}(x, s)= \begin{cases}\phi_{i}(|s|) & g(x, s) \leq i \\ \frac{i \phi_{i}(|s|)}{g(x, s)} & g(x, s)>i\end{cases}
$$

and define

$$
g_{i}(x, s, z)=\eta_{i}(x, s) f(x, s, z)+\left(1-\eta_{i}(x, s)\right) c_{0} \Phi(|z|) .
$$

The functions $g_{i}$ are Carathéodory functions, quasi-convex with respect to $z$ such that

$$
\begin{gathered}
c_{0} \Phi(|z|) \leq g_{i}(x, s, z) \leq\left(i+c_{0}\right)(1+\Phi(|z|)), \\
g_{i}(x, s, z)=c_{0} \Phi(|z|) \quad|s| \geq i, \\
g_{i}(x, s, z)=f(x, s, z) \quad i>g(x, s)+|s|+1, \\
\lim _{i} g_{i}(x, u, z)=\sup _{i} g_{i}(x, s, z)=f(x, s, z) .
\end{gathered}
$$

Now, we perform a truncation with respect to $z$. Define the Carathéodory function

$$
g_{i m}(x, s, z)=\phi_{m}(|z|) g_{i}(x, s, z)+\left(1-\phi_{m}(|z|)\right) c_{0} \Phi(|z|) .
$$

The functions $g_{i m}$ are not quasi-convex with respect to $z$, therefore we consider their quasi-convex envelopes $G_{i m}$. Hence, the quasi-convexity of $G_{i m}$ and condition $(3.17)_{1}$ imply

$$
c_{0} \Phi(|z|) \leq G_{i m}(x, s, z) \leq\left(i+c_{0}\right)(1+\Phi(|z|)),
$$

moreover, $(3.17)_{2}$ yields

$$
G_{i m}(x, s, z)=c_{0} \Phi(|z|)
$$

for $|z| \geq m,|s| \geq i$. By taking into account (3.19) and (3.20), we may apply Theorem 2.6 and find the following integral representation formula for $G_{i m}$ :

$$
G_{i m}(x, s, z)=\inf \left\{f_{\Omega} g_{i m}(x, s, z+D \varphi(y)) d y: \varphi \in W_{0}^{1} E^{\Phi}\left(\Omega, \mathbb{R}^{N}\right)\right\} .
$$

The more significant and technically difficult part of the proof is worked out in Lemma 3.3 below in which we prove that $\left(G_{i m}\right)_{m \in \mathbb{N}}$ converges to $g_{i}$ pointwise on $\Omega$. Assuming Lemma 3.3 holds true, we can conclude the proof of Theorem 3.2. Indeed, for $k \geq 2+c_{0}$ define

$$
f_{k}(x, s, z)=\max \left\{G_{i m}(x, s, z): i+m \leq k\right\},
$$

then $f_{k}$ satisfies (3.14), (3.15) and (3.16). 
Lemma 3.3. For every $i \in \mathbb{N}$ the sequence $\left(G_{i m}\right)_{m \in \mathbb{N}}$ converges to $g_{i}$ pointwise on $\Omega$.

Proof. With fixed $i \in \mathbb{N}$ let $\left(x_{0}, s_{0}, z_{0}\right) \in \Omega \times \mathbb{R}^{N} \times \mathbb{R}^{N n}$. First notice that $\left(G_{i m}\left(x_{0}, s_{0}, z_{0}\right)\right)_{m \in \mathbb{N}}$ is a non-decreasing sequence and that for every $m \in \mathbb{N}$ inequality $G_{i m}\left(x_{0}, s_{0}, z_{0}\right) \leq g_{i}\left(x_{0}, s_{0}, z_{0}\right)$ is trivial by the very definition of $G_{i m}$ (see (3.18) and (3.21)).

By the representation formula (3.21), for fixed $m \in \mathbb{N}$ there exists $w_{m} \in$ $W_{0}^{1} E^{\Phi}\left(\Omega, \mathbb{R}^{N}\right)$ such that

$$
f_{\Omega} g_{i m}\left(x_{0}, s_{0}, z_{0}+D w_{m}\right) d y \leq G_{i m}\left(x_{0}, s_{0}, z_{0}\right)+\frac{1}{m} .
$$

Consider the functional

$$
v \in W_{0}^{1,1}\left(\Omega, \mathbb{R}^{N}\right) \rightarrow f_{\Omega} g_{i m}\left(x_{0}, s_{0}, z_{0}+D v\right) d y,
$$

by taking into account Ekeland's Variational Principle (see [14], [24]) there exists $u_{m} \in W_{0}^{1,1}\left(\Omega, \mathbb{R}^{N}\right)$ such that

$$
f_{\Omega} g_{i m}\left(x_{0}, s_{0}, z_{0}+D u_{m}\right) d y \leq G_{i m}\left(x_{0}, s_{0}, z_{0}\right)+\frac{1}{m},
$$

and such that for every $\varphi \in W_{0}^{1,1}\left(\Omega, \mathbb{R}^{N}\right)$ there holds

$$
\begin{aligned}
& f_{\Omega} g_{i m}\left(x_{0}, s_{0}, z_{0}+D u_{m}\right) d y \\
& \quad \leq f_{\Omega} g_{i m}\left(x_{0}, s_{0}, z_{0}+D \varphi\right) d y+\frac{1}{m} \int_{\Omega}\left|D u_{m}-D \varphi\right| d y .
\end{aligned}
$$

Let us prove that $\left(u_{m}\right)$ is a sequence of $Q$-minima, with $Q$ independent on $m$, of the functional

$$
v \in W_{0}^{1} E^{\Phi}\left(\Omega, \mathbb{R}^{N}\right) \rightarrow \int_{\Omega}(1+\Phi(|D v|)) d y,
$$

i.e., there exists $Q$ such that for every $\varphi \in W_{0}^{1} E^{\Phi}\left(\Omega, \mathbb{R}^{N}\right)$, denoted $\Sigma=\operatorname{supp}\left(\varphi-u_{m}\right)$, there holds

$$
\int_{\Sigma}\left(1+\Phi\left(\left|D u_{m}\right|\right)\right) d y \leq Q \int_{\Sigma}(1+\Phi(|D \varphi|)) d y .
$$

First notice that formulas $(3.17)_{1},(3.19)$ and (3.23) yield, for $m$ sufficiently large,

$$
\begin{aligned}
& c_{0} \int_{\Sigma} \Phi\left(\left|z_{0}+D u_{m}\right|\right) d y \\
& \quad \leq\left(i+c_{0}\right) \int_{\Sigma}\left(1+\Phi\left(\left|z_{0}+D \varphi\right|\right)\right) d y+\int_{\Sigma}\left|D u_{m}-D \varphi\right| d y .
\end{aligned}
$$


Without loss of generality we may assume $c_{0} \leq 1$, hence Young's inequality yields

$$
\left|D u_{m}-D \varphi\right| \leq \frac{c_{0}}{2^{r}} \Phi\left(\left|D u_{m}\right|\right)+\frac{c_{0}}{2^{r}} \Phi(|D \varphi|)+\widetilde{\Phi}\left(\frac{2^{r}}{c_{0}}\right),
$$

then combining (3.25), (3.26) and assumption $\Phi \in \Delta_{2}^{r}$ we get

$$
\begin{aligned}
& \int_{\Sigma} \Phi\left(\left|D u_{m}\right|\right) d y \\
& \quad \leq\left(\frac{i}{c_{0}}+2\right) \int_{\Sigma} \Phi(|D \varphi|) d y+4^{r}\left(\Phi\left(\left|z_{0}\right|\right)+\widetilde{\Phi}\left(\frac{2^{r}}{c_{0}}\right)+\frac{i}{c_{o}}+1\right) \mathcal{L}^{n}(\Omega),
\end{aligned}
$$

which implies (3.24) with $Q=Q\left(c_{0}, r, i, \Phi\left(\left|z_{0}\right|\right)\right)$.

Let $\Omega_{0} \subset \subset \Omega$ be fixed, formulas (3.19) and (3.22) give

$$
c_{0} f_{\Omega} \Phi\left(\left|z_{0}+D u_{m}\right|\right) d y \leq\left(i+c_{0}\right)\left(1+\Phi\left(\left|z_{0}\right|\right)\right),
$$

hence, setting $\Omega_{m}=\left\{y \in \Omega_{0}:\left|z_{0}+D u_{m}(y)\right|>m-1\right\}$, we get

$$
\Phi(m-1) \mathcal{L}^{n}\left(\Omega_{m}\right) \leq\left(\frac{i}{c_{0}}+1\right)\left(1+\Phi\left(\left|z_{0}\right|\right)\right) \mathcal{L}^{n}(\Omega),
$$

and then $\mathcal{L}^{n}\left(\Omega_{m}\right) \rightarrow 0$ for $m \rightarrow+\infty$.

Moreover, by (3.19) and (3.22) it follows that

$$
\sup _{m} \int_{\Omega} \Phi\left(\left|D u_{m}\right|\right) d y<+\infty,
$$

hence by taking into account the regularity result of Theorem 2.7, we have that there exists $\delta>0$, independent on $m$, such that

$$
\sup _{m} \int_{\Omega_{0}} \Phi\left(\left|D u_{m}\right|\right)\left(\frac{\Phi\left(\left|D u_{m}\right|\right)}{\left|D u_{m}\right|}\right)^{\delta} d y<+\infty .
$$

Define $\Phi_{\delta}(t)=\Phi(t)\left[\frac{\Phi(t)}{t}\right]^{\delta}$ and let $\Gamma_{\delta}(s)=\Phi_{\delta}\left(\Phi^{-1}(s)\right)$, the function

$$
\Psi_{\delta}(t)=\int_{0}^{t} \frac{\Gamma_{\delta}(s)}{s} d s
$$

is an $\mathrm{N}$-function of class $\Delta_{2}$ such that $\Psi_{\delta}(t) \leq \Gamma_{\delta}(t)$ for every $t \in[0,+\infty)$. Hence, by taking into account Young's inequality we get

$$
\int_{\Omega_{m}} \Phi\left(\left|D u_{m}\right|\right) d y \leq\left\|\Phi\left(\left|D u_{m}\right|\right)\right\|_{L^{\Psi_{\delta}\left(\Omega_{m}\right)}}\left\|1_{\Omega_{m}}\right\|_{L^{\Psi_{\delta}\left(\Omega_{m}\right)}} .
$$

Notice that

$$
\left\|1_{\Omega_{m}}\right\|_{L^{\Psi_{\delta}\left(\Omega_{m}\right)}}=\mathcal{L}^{n}\left(\Omega_{m}\right) \Psi_{\delta}^{-1}\left(\frac{1}{\mathcal{L}^{n}\left(\Omega_{m}\right)}\right),
$$


and thus we infer $\left\|1_{\Omega_{m}}\right\|_{L^{\Psi_{\delta}\left(\Omega_{m}\right)}} \rightarrow 0$ for $m \rightarrow+\infty$. Moreover, the very definition of the Orlicz norm yields

$$
\left\|\Phi\left(\left|D u_{m}\right|\right)\right\|_{L^{\Psi_{\delta}\left(\Omega_{m}\right)}} \leq 1+\int_{\Omega_{m}} \Gamma_{\delta}\left(\Phi\left(\left|D u_{m}\right|\right)\right) d y,
$$

and therefore by (3.28) we can conclude

$$
\lim _{m} \int_{\Omega_{m}} \Phi\left(\left|D u_{m}\right|\right) d y=0 .
$$

By the generalization of the Poincaré inequality to $\mathrm{N}$-functions (see Lemma 5.7 of [24]) and (3.27) the sequence $\left(u_{m}\right) \subset W_{0}^{1} E^{\Phi}\left(\Omega, \mathbb{R}^{N}\right)$ has equibounded norms in $W^{1} L^{\Phi}\left(\Omega, \mathbb{R}^{N}\right)$. Therefore, there exists a subsequence, still denoted by $\left(u_{m}\right)$, converging to a function $u \in W_{0}^{1} E^{\Phi}\left(\Omega, \mathbb{R}^{N}\right)$ in $* w-W^{1} L^{\Phi}\left(\Omega, \mathbb{R}^{N}\right)$.

Consider inequality (3.22), then the choice of $\Omega_{m}$ and formulas (3.17) 1 , (3.18) yield

$$
\begin{aligned}
& G_{i m}\left(x_{0}, s_{0}, z_{0}\right)+\frac{1}{m} \geq \frac{1}{\mathcal{L}^{n}(\Omega)} \int_{\Omega_{0} \backslash \Omega_{m}} g_{i}\left(x_{0}, s_{0}, z_{0}+D u_{m}\right) d y \\
& \geq \frac{1}{\mathcal{L}^{n}(\Omega)} \int_{\Omega_{0}} g_{i}\left(x_{0}, s_{0}, z_{0}+D u_{m}\right) d y-\frac{i+c_{0}}{\mathcal{L}^{n}(\Omega)} \int_{\Omega_{m}}\left(1+\Phi\left(\left|z_{0}+D u_{m}\right|\right)\right) d y .
\end{aligned}
$$

Hence, by taking into account the lower semicontinuity result in the autonomous case $f=f(z)$ (see Theorem 3.1 of [15]) and formula (3.29), passing to the limit in (3.30) we have

$$
\begin{aligned}
& \lim _{m} G_{i m}\left(x_{0}, s_{0}, z_{0}\right) \\
& \geq \int_{\Omega} g_{i}\left(x_{0}, s_{0}, z_{0}+D u\right) d y-\frac{1}{\mathcal{L}^{n}(\Omega)} \int_{\Omega \backslash \Omega_{0}} g_{i}\left(x_{0}, s_{0}, z_{0}+D u\right) d y \\
& \geq g_{i}\left(x_{0}, s_{0}, z_{0}\right)-\frac{1}{\mathcal{L}^{n}(\Omega)} \int_{\Omega \backslash \Omega_{0}} g_{i}\left(x_{0}, s_{0}, z_{0}+D u\right) d y,
\end{aligned}
$$

where the last inequality follows by the quasi-convexity of $g_{i}$. Indeed, condition $(3.17)_{1}$ assures the continuity of the functional

$$
v \in W^{1} L^{\Phi}\left(\Omega, \mathbb{R}^{N}\right) \rightarrow \int_{\Omega} g_{i}(x, v, D v) d x
$$

in the strong topology of $W^{1} L^{\Phi}\left(\Omega, \mathbb{R}^{N}\right)$, thus quasi-convexity inequality for $g_{i}$ can also be extended to test functions in $W_{0}^{1} E^{\Phi}\left(\Omega, \mathbb{R}^{N}\right)$.

Eventually, by letting $\mathcal{L}^{n}\left(\Omega \backslash \Omega_{0}\right) \rightarrow 0$ in (3.31) we get

$$
\lim _{m} G_{i m}\left(x_{0}, s_{0}, z_{0}\right) \geq g_{i}\left(x_{0}, s_{0}, z_{0}\right),
$$

which concludes the proof of the lemma.

We can now prove Theorem 3.1. 
Proof of Theorem 3.1. With fixed $\varepsilon>0$, define

$$
f_{\varepsilon}(x, s, z)=f(x, s, z)+c_{2} \Phi_{2}(|s|)+c_{3}(x)+\varepsilon \Phi(|z|)+c_{\varepsilon},
$$

where $c_{\varepsilon}>0$ is chosen such that

$$
f_{\varepsilon}(x, s, z) \geq \frac{\varepsilon}{2} \Phi(|z|),
$$

for every $(x, s, z) \in \Omega \times \mathbb{R}^{N} \times \mathbb{R}^{N n}$. The existence of such $c_{\varepsilon}$ follows by the growth conditions (3.11) of $f$ and the assumption $\Phi_{1} \prec \prec \Phi$.

Let $\left(f_{\varepsilon, k}\right)_{k}$ be the sequence of quasi-convex functions provided by Theorem 3.2, then arguing as in Lemma 4.3 of [32] the functionals $F_{\varepsilon}^{k}: W^{1} L^{\Phi}\left(\Omega, \mathbb{R}^{N}\right) \rightarrow \mathbb{R}$ defined by

$$
F_{\varepsilon}^{k}(u, \Omega)=\int_{\Omega} f_{\varepsilon, k}(x, u, D u) d x
$$

are sequentially lower semicontinuous in $* w-W^{1} L^{\Phi}\left(\Omega, \mathbb{R}^{N}\right)$. Moreover, consider the functional $F_{\varepsilon}: W^{1} L^{\Phi}\left(\Omega, \mathbb{R}^{N}\right) \rightarrow \mathbb{R}$ defined by

$$
F_{\varepsilon}(u, \Omega)=\int_{\Omega} f_{\varepsilon}(x, u, D u) d x,
$$

since $F_{\varepsilon}(\cdot, \Omega)=\sup _{k} F_{\varepsilon}^{k}(\cdot, \Omega)$ we infer the lower semicontinuity of $F_{\varepsilon}(\cdot, \Omega)$ in $* w-W^{1} L^{\Phi}\left(\Omega, \mathbb{R}^{N}\right)$.

Let $\left(u_{r}\right)$ be a sequence weakly* converging to $u$ in $W^{1} L^{\Phi}\left(\Omega, \mathbb{R}^{N}\right)$, since the choice of $\Phi_{2}$, Proposition 2.4 and the embedding Theorem 2.5 yield the convergence of $\left(u_{r}\right)$ to $u$ in the norm topology of $L^{\Phi_{2}}\left(\Omega, \mathbb{R}^{N}\right)$ and $u \in E^{\Phi_{2}}\left(\Omega, \mathbb{R}^{N}\right)$. Hence, by applying Proposition 2.3 we infer

$$
\lim _{r} \int_{\Omega} \Phi_{2}\left(\left|u_{r}\right|\right) d x=\int_{\Omega} \Phi_{2}(|u|) d x,
$$

and since the $* w-W^{1} L^{\Phi}\left(\Omega, \mathbb{R}^{N}\right)$ convergence of $\left(u_{r}\right)$ to $u$ yields

$$
\sup _{r} \int_{\Omega} \Phi\left(\left|D u_{r}\right|\right) d x \leq M,
$$

we get

$$
\begin{aligned}
& \liminf _{r} \int_{\Omega} f\left(x, u_{r}, D u_{r}\right) d x \\
& \geq \liminf _{r} \int_{\Omega} f_{\varepsilon}\left(x, u_{r}, D u_{r}\right) d x-\int_{\Omega}\left(c_{2} \Phi_{2}(|u|)+c_{3}(x)+c_{\varepsilon}\right) d x-\varepsilon M \\
& \geq \int_{\Omega} f_{\varepsilon}(x, u, D u) d x-\int_{\Omega}\left(c_{2} \Phi_{2}(|u|)+c_{3}(x)+c_{\varepsilon}\right) d x-\varepsilon M \\
& \geq \int_{\Omega} f(x, u, D u) d x-\varepsilon M,
\end{aligned}
$$

which concludes the proof as $\varepsilon \rightarrow 0$. 


\section{Existence and applications}

Let us first recall a few facts about the trace operator in Orlicz-Sobolev spaces. Let $\Omega$ be such that $\partial \Omega$ is Lipschitz regular, in this case one can define a trace operator from $W^{1} E^{\Phi}\left(\Omega, \mathbb{R}^{N}\right)$ to $E^{\Phi}\left(\partial \Omega, \mathbb{R}^{N}\right)$ whose kernel is exactly $W_{0}^{1} E^{\Phi}\left(\Omega, \mathbb{R}^{N}\right)$. Note that in the case $\Phi \in \Delta_{2}$ we have $W^{1} L^{\Phi}\left(\Omega, \mathbb{R}^{N}\right) \equiv W^{1} E^{\Phi}\left(\Omega, \mathbb{R}^{N}\right)$, $L^{\Phi}\left(\partial \Omega, \mathbb{R}^{N}\right) \equiv E^{\Phi}\left(\partial \Omega, \mathbb{R}^{N}\right)$ and $W_{0}^{1} E^{\Phi}\left(\Omega, \mathbb{R}^{N}\right)$ is $* w-W^{1} L^{\Phi}\left(\Omega, \mathbb{R}^{N}\right)$ closed (see [20], [28]).

The last statement enables us to consider Dirichlet's boundary values problems in Orlicz-Sobolev spaces in case $\Phi \in \Delta_{2}$.

In the vectorial setting, as pointed out in [24], the most natural growth conditions to impose on the stored energy densities $f$ are the ones given below, i.e.,

$$
\begin{aligned}
-c_{1} \Phi_{1}(|z|)-b(x) \Phi_{2}(|s|)-c_{3}(x) & \leq f(x, s, z) \\
& \leq c_{2} \Phi(|z|)+b(x) \Phi_{2}(|s|)+c_{3}(x), \\
f(x, s, z) \geq \tilde{f}(z) & -b(x) \Phi_{2}(|s|)-c_{3}(x),
\end{aligned}
$$

where in (4.32) $c_{i}>0$ for $i=1,2, c_{3} \in L^{1}(\Omega), \Phi, \Phi_{1}$ and $\Phi_{2}$ are $\mathrm{N}$-functions such that $\Phi \in \Delta_{2}$ and $\Phi_{i} \prec \prec$ for $i=1,2$. Moreover, in (4.33) $b \in E^{\widetilde{\Gamma}}(\Omega)$ with $\Gamma$ defined by (2.6) is such that $\Gamma \circ \Phi_{2} \prec \Phi$ and $\tilde{f}: \mathbb{R}^{N n} \rightarrow \mathbb{R}$ is a strictly quasi-convex function in $z=0$, i.e., $\widetilde{f}$ is a continuous function such that

$$
c_{4} \int_{\Omega} \Phi(|D \varphi|) d x+\widetilde{f}(0) \mathcal{L}^{n}(\Omega) \leq \int_{\Omega} \tilde{f}(D \varphi) d x
$$

for every $\varphi \in W_{0}^{1} E^{\Phi}\left(\Omega, \mathbb{R}^{N}\right)$ with $c_{4}>0$. Without loss of generality we may also assume $\tilde{f}(0)=0$.

Let us state and prove the following existence result (for related results in the poly-convex case see [4]):

Theorem 4.1. Let $f: \Omega \times \mathbb{R}^{N} \times \mathbb{R}^{N n} \rightarrow[0,+\infty)$ be a Carathéodory function, quasi-convex with respect to $z$ satisfying (4.32), (4.33).

Let $u_{0} \in W^{1} L^{\Phi}\left(\Omega, \mathbb{R}^{N}\right)$, consider the Dirichlet's class

$$
V_{0}=u_{0}+W_{0}^{1} E^{\Phi}\left(\Omega, \mathbb{R}^{N}\right)
$$

then the variational problem

$$
\inf \left\{F(u, \Omega): u \in V_{0}\right\}
$$

has a solution.

Proof. Let $u \in V_{0}$ and set $\varphi=\left(u-u_{0}\right)$, then the assumption $\Phi \in \Delta_{2}$ and formulas (4.33), (4.34) yield

$$
\begin{aligned}
& \int_{\Omega} \Phi(|D u|) d x \leq c \int_{\Omega} \Phi(|D \varphi|) d x+c \int_{\Omega} \Phi\left(\left|D u_{0}\right|\right) d x \\
& \quad \leq c \int_{\Omega} \tilde{f}(D \varphi) d x+c \int_{\Omega} \Phi\left(\left|D u_{0}\right|\right) d x \\
& \quad \leq c \int_{\Omega} f(x, u, D \varphi) d x+c \int_{\Omega}\left(b(x) \Phi_{2}(|u|)+c_{3}(x)+\Phi\left(\left|D u_{0}\right|\right)\right) d x
\end{aligned}
$$


denoting with $c$ a generic constant which may vary from line to line. By taking into account Proposition 3.2 of [15] we have

$$
\begin{aligned}
& \int_{\Omega} f(x, u, D \varphi) d x \\
& \quad=\int_{\Omega}(f(x, u, D \varphi)-f(x, u, D u)) d x+\int_{\Omega} f(x, u, D u) d x \\
& \leq c \int_{\Omega}\left(p(|\theta|)+p(|D u|)+p\left(\left|D u_{0}\right|\right)\right)\left|D u_{0}\right| d x+\int_{\Omega} f(x, u, D u) d x
\end{aligned}
$$

where $\theta(x)=\Phi^{-1}\left(c_{3}(x)+b(x) \Phi_{2}(|u(x)|)\right)$ and recall that $p$ is the right derivative of $\Phi$. Notice that since $b \in E^{\widetilde{\Gamma}}(\Omega)$ then, by Young's inequality,

$$
\int_{\Omega} b(x) \Phi_{2}(|u|) d x \leq \int_{\Omega} \widetilde{\Gamma}\left(\frac{1}{\varepsilon}|b(x)|\right) d x+\varepsilon \int_{\Omega} \Phi(|u|) d x,
$$

which implies that $\theta \in L^{\Phi}\left(\Omega, \mathbb{R}^{N}\right)$. Moreover, by taking into account assumption $\Phi \in \Delta_{2}$, (4.37), Young's inequality and Poincaré's inequality for $\mathrm{N}$-functions it follows that

$$
\begin{aligned}
& \int_{\Omega}\left(p(|\theta|)+p(|D u|)+p\left(\left|D u_{0}\right|\right)\right)\left|D u_{0}\right| d x \\
& \quad \leq c \int_{\Omega}\left(c_{3}(x)+\Phi\left(\left|D u_{0}\right|\right)+\widetilde{\Gamma}\left(c_{\varepsilon}|b(x)|\right)\right) d x+\varepsilon \int_{\Omega}(\Phi(|u|)+\Phi(|D u|)) d x \\
& \quad \leq c \int_{\Omega}\left(c_{3}(x)+\Phi\left(\left|u_{0}\right|\right)+\Phi\left(\left|D u_{0}\right|\right)+\widetilde{\Gamma}\left(c_{\varepsilon}|b(x)|\right)\right) d x+2 \varepsilon \int_{\Omega} \Phi(|D u|) d x .
\end{aligned}
$$

Hence, collecting (4.35), (4.36) and (4.38) we get

$$
\begin{aligned}
& \int_{\Omega} \Phi(|D u|) d x \leq c \int_{\Omega} f(x, u, D u) d x \\
& \quad+c \int_{\Omega}\left(c_{3}(x)+\Phi\left(\left|u_{0}\right|\right)+\Phi\left(\left|D u_{0}\right|\right)+\widetilde{\Gamma}\left(c_{\varepsilon}|b(x)|\right)\right) d x,
\end{aligned}
$$

which yield the coercivity of $F(\cdot, \Omega)$ on $V_{0}$.

Eventually, by applying Theorem 3.1, the Direct Methods yield the existence of a minimizer for $F(\cdot, \Omega)$ on $V_{0}$.

We now give some applications of our result.

Zhang in [37] developed a method to construct non-trivial, i.e., non-convex, quasi-convex functions $g_{p}=g_{p}(z)$ with polynomial growth $p \geq 1$ at infinity. Under additional assumptions the resulting functions $g_{p}$ are not even poly-convex.

In [15], [16] a suitable modification of Zhang's method, i.e., using N-functions instead of powers, enabled the construction of quasi-convex functions $g_{\Phi}=g_{\Phi}(z)$ satisfying the non-standard growth conditions

$$
c_{0}(\Phi(|z|)-1) \leq g_{\Phi}(z) \leq c_{1}(\Phi(|z|)+1)
$$


with $c_{0}, c_{1}>0$. Therefore, given a function $a \in L^{\infty}\left(\Omega \times \mathbb{R}^{N}\right)$ such that $a(x, s) \geq$ $c_{2}>0$ a.e., the function $f_{\Phi}(x, s, z)=a(x, s) g_{\Phi}(z)$ satisfies conditions (4.32), (4.33) of the existence Theorem 4.1. Hence, we may apply the result above to solve Dirichlet's boundary values problems for the integral functionals

$$
F_{\Phi}(u, \Omega)=\int_{\Omega} f_{\Phi}(x, u, D u) d x .
$$

We remark that in the case of variational integrals whose integrands $f$ have $(p, q)$ growth $(p<q)$ and depend on the full set of variables, weak lower semicontinuity results in $W^{1, p}\left(\Omega, \mathbb{R}^{N}\right)$ are available only under additional continuity assumptions on the dependence of $f$ on $(x, s)$ (see Remark 4.3 of [17]). Moreover, there is a restriction on the mutual dependence of $p$ and $q$. Our approach bypasses these limitations for functionals whose energy densities are controlled in terms of $\mathrm{N}$-functions of class $\Delta_{2}$.

Let, for instance, $\Phi(t)=\frac{t^{2}}{\log (1+t)}$, then the corresponding $f_{\Phi}$ has $(p, q)$ growth with $p=2-\varepsilon$ and $q=2$ for every $\varepsilon>0$. The known results in ordinary Sobolev spaces imply the sequential lower semicontinuity of $F_{\Phi}(\cdot, \Omega)$ in the weak topology of $W^{1,2}\left(\Omega, \mathbb{R}^{N}\right)$, but the functional is coercive only on $W^{1,2-\varepsilon}\left(\Omega, \mathbb{R}^{N}\right)$. Thus, the Sobolev spaces setting does not allow the use of the Direct Methods to solve Dirichlet's boundary value problems unless one is able to exhibit a minimizing sequence possessing higher integrability properties.

Eventually, let $\Phi(t)=t^{a+b \sin (\sin (\log (t)))}$ with $a>1+b \sqrt{2}$, and let $f_{\Phi}=$ $f_{\Phi}(x, z)$. Notice that $f_{\Phi}$ has $(p, q)$ growth with $p=a-b$ and $q=a+b$, thus the results of Marcellini [33] ensures the weak lower semicontinuity of $F_{\Phi}(\cdot, \Omega)$ in $W^{1, a-b}\left(\Omega, \mathbb{R}^{N}\right)$ provided $a>(2 n+1) b$ (see the introduction), that is when $p, q$ are relatively close to each other, i.e., $q-p<\frac{a-b}{n}$. On the other hand, Theorem 3.1 does not impose any further restriction on $a$ and $b$, and thus Theorem 4.1 can be applied to solve Dirichlet's boundary value problems for any $a, b$ chosen as above.

\section{References}

1. Acerbi, E., Dal Maso, G.: New lower semicontinuity results for polyconvex integrals case. Calc. Var. 2, 329-372 (1994)

2. Acerbi, E., Fusco, N.: Semicontinuity problems in the calculus of variations. Arch. Rat. Mech. Anal. 86, 125-145 (1984)

3. Adams, R.A.: Sobolev Spaces. New York: Academic Press 1975

4. Ball, J.M.: Convexity conditions and existence theorems in nonlinear elasticity. Arch. Rat. Mech. Anal. 63, 337-403 (1977)

5. Celada, P., Dal Maso, G.: Further remarks on the lower semicontinuity of polyconvex integrals. Ann. Inst. H. Poincaré (Anal. non Linéarie) 11, 661-691 (1995)

6. Cianchi, A.: A sharp embedding theorem for Orlicz-Sobolev spaces. Indiana Univ. Math. J. 45, 39-65 (1996)

7. Cianchi, A., Fusco, N.: Gradient regularity for minimizers under general growth conditions. J. Reine Angew. Math. 507, 15-36 (1999)

8. Dacorogna, B.: Direct methods in the calculus of variations. Appl. Math. Sci. 78, Heidelberg, New York: Springer 1989

9. Dacorogna, B., Marcellini, P.: Semicontinuité pour des intégrandes polyconvexes sans continuité des déterminants. C.R.Acad. Sci. Paris 311, Série I, 393-396 (1990) 
10. Dall'Aglio, A., Mascolo, E., Papi, G.: Regularity for local minima of functionals with non-standard growth conditions. Rend. Mat. 18, Serie VII, Roma 305-326 (1998)

11. Dal Maso, G., Sbordone, C.: Weak lower semicontinuity of polyconvex integrals: a borderline case. Math. Z. 218, 603-609 (1995)

12. De Giorgi, E.: Teoremi di semicontinuità nel Calcolo delle Variazioni. Roma: INdAM 1968-69

13. Donaldson, T.K., Trudinger, N.S.: Orlicz-Sobolev spaces and embedding theorems. J. Funct. Anal. 8, 42-75 (1971)

14. Ekeland, I.: On the Variational Principle. J. Math. Anal. Appl. 47, 324-353 (1974)

15. Focardi, M.: Semicontinuity of vectorial functionals in Orlicz-Sobolev spaces. Rend. Ist. Mat. Univ. Trieste XXIX, 141-161 (1997)

16. Focardi, M., Mascolo, E.: Lower semicontinuity of quasi-convex functionals with non standard growth. Journal of Convex Analysis 8, 1-22 (2001)

17. Fonseca, I., Malý, J.: Relaxation of multiple integrals in Sobolev spaces below the growth exponent for the energy density. Ann. Inst. H. Poincaré (Analyse non Linéaire) 14, 309-338 (1997)

18. Fonseca, I., Marcellini, P.: Relaxation of multiple integrals in subcritical Sobolev spaces. J. Geom. Anal. 7, 57-81 (1997)

19. Fonseca, I., Müller, S.: Quasi-convex integrands and lower semicontinuity in $L^{1}$. SIAM J. Math. Anal. 23, 1081-98 (1992)

20. Fougères, A.: Théorèmes de trace et de prolongement dans les espaces de Sobolev et de Sobolev-Orlicz. C.R. Acad. Sci. Paris Série A 274, 181-184 (1972)

21. Fusco, N.: Quasi-convessità e semicontinuità per integrali multipli di ordine superiore. Ricerche di Mat. 29, 307-323 (1980)

22. Fusco, N., Hutchinson, J.E.: A direct proof of lower semicontinuity of polyconvex functionals. Manuscripta Mat. 87, 35-50 (1995)

23. Gangbo, W.: On the weak lower semicontinuity of energies with polyconvex integrands. J. Math Pures Appl. 73, 5 (1994)

24. Giusti, E.: Metodi diretti nel Calcolo delle Variazioni. Bologna: U.M.I. 1994

25. Gossez, J.P.: Nonlinear elliptic problems for equations with rapidly (or slowly) increasing coefficients. Trans. Amer. Math Soc. 55, 163-205 (1974)

26. Krasnosel'skij, M.A., Rutickii, Ya.B.: Convex functions and Orlicz spaces. Groningen: Noordhoff 1961

27. Kristensen, J.: Lower semicontinuity in Sobolev spaces below the growth exponent of the integrand. Proc. Roy. Soc. Edinburgh, Sect. A 127, 797-817 (1997)

28. Kufner, A., John, O., Fučik, S.: Function Spaces. Praha: Academia 1977

29. Malý, J.: Weak lower semicontinuity of polyconvex integrals. Proc. Roy. Soc. Edinburgh, Sect. A 123, 681-691 (1993)

30. Malý, J.: Weak lower semicontinuity of polyconvex and quasi-convex integrals. Bonn: Vortagsreihe 1993

31. Malý, J.: Lower semicontinuity of quasi-convex integrals. Manus. Math. 85, 419-428 (1994)

32. Marcellini, P.: Approximation of quasi-convex functions and lower semicontinuity of multiple integrals. Manus.Math. 51, 1-28 (1985)

33. Marcellini, P.: On the definition and the lower semicontinuity of certain quasi-convex integrals. Ann. Inst. H. Poincaré (Analyse non Linéaire) 3, 391-409 (1986)

34. Morrey, C.B., Jr.: Quasi-convexity and the lower semicontinuity of multiple integrals. Pacific J. Math. 2, 25-53 (1952)

35. Rao, M.M., Ren, Z.D.: Theory of Orlicz spaces. Pure and Appl. Math., New York: Marcel Dekker 1981

36. Trudinger, N.S.: On imbeddings into Orlicz spaces and some applications. J. Math. Mech. 17, 473-483 (1967)

37. Zhang, K.: A construction of quasi-convex functions with linear growth at infinity. Ann. Scuola Norm. Sup. Pisa 19, 313-326 (1992)

38. Ziemer, W.: Weakly Differentiable Functions. GTM 120, Springer 1989 